\title{
ON FROBENIUS EXTENSIONS II
}

\author{
TADASI NAKAYAMA and TOSIRO TSUZUKU
}

In Part $\mathrm{I}^{1)}$ we introduced the notion of 2 . Frobenius extensions of a ring, as a generalization of Kasch's [10] Frobenius extensions and hence of classical Frobenius algebras. We proved, in I, bilinear (or sesqui-linear, rather, to follow Bourbaki's terminology) form and scalar product characterizations of Frobenius extensions in such extended sense, generalizing Kasch's and classical case, and then studied homological dimensions in them, generalizing and refining the results in Eilenberg-Nakayama [4] and Hirata [6]. Dual bases were considered in case of quasi-free (2.) Frobenius extensions Also the case of a semi-primary or S-ring ground ring was studied.

In the present Part II we continue our study of Frobenius extensions in such generalized sense. Thus we first study relative homological dimensions in them, generalizing the Maschke-Ikeda-Kasch characterization of relatively projective and relatively injective modules as well as Hirata's [6] results. Then in $\$ 7$ we establish Kasch's [10] theorem on the endomorphism ring of a Frobenius ring for our generalized case. Here the removal of Kasch's S-ring assumption (which we have already discussed in our previous note [13]) and the replacement of free module property with projective or quasi-free ones make our proof more complicated, respectively in substance and in computation, than Kasch's case.

Then, in $\S 8$, we transfer to the present case the annihilator relations given in [17] for classical Frobenius algebras, on restricting ourselves to $d$-ideals (similar to (but slightly more general than) $v$-ideals in Kasch [10]). Further, in 9 we consider residue-rings of a Frobenius extension, in order to study when they are also Frobenius extensions. In these considerations Frobenius extensions are naturally taken in our generalized sense and thus deviations, some rather essential and some rather formal, from (the classical case and)

Received May 12, 1961.

1) T. Nakayama-T. Tsuzuku, On Frobenius extensions I, Nagoya Math. J. 17 (1960), 89-110. 
Kasch's [10] case are needed. In $\S 10$ the case of a quasi-Frobenius ground ring is considered. Thus, Hall's [15] theorem (on the ordinary scalar product in vector-modules over a quasi-Frobenius ring) is observed to be transferable to the case of a regular sesqui-linear scalar product in projective modules, and this is combined with $\S 9$ to consider residue-rings of (quasi-Frobenius) Frobenius extensions of a quasi-Frobenius ring.

\section{§ 6. Relative homological dimensions in Frobenius dimensions.}

First we proceed in parallel with $\mathrm{I}, \S 3$, generalizing Hirata [6]. Let $A$ be a ring (with unit element) and $B$ a subring of $A$ (containing the unit element of $A$ ).

Lemma 17. Let $\mathfrak{Q}$ be an A-left-module and $\mathrm{m}$ a B-left-module. Then

$$
\begin{gathered}
\operatorname{Ext}_{(A, B)}^{q}\left(\mathfrak{L}, A \otimes_{B} \mathrm{~m}\right)= \begin{cases}0 & q>0, \\
\operatorname{Hom}_{B}\left(\mathfrak{L},\left(\beta^{-1}, \mathfrak{m}\right)\right) & q=0,\end{cases} \\
\operatorname{Ext}_{(A, B)}^{q}\left(\operatorname{Hom}_{B}(A, \mathrm{~m}), \mathfrak{L}\right)= \begin{cases}0 & q>0 \\
\operatorname{Hom}_{B}\left(\left(\beta^{-1}, \mathrm{~m}\right), \mathfrak{l}\right) & q=0,\end{cases}
\end{gathered}
$$

Let further $\mathfrak{R}$ be an A-right-module. Then

$$
\operatorname{Tor}_{(A, B)}^{q}\left(\mathfrak{R}, \operatorname{Hom}_{B}(A, \mathrm{~m})\right)= \begin{cases}0 & q>0 \\ \Re \otimes_{B}(\beta, \mathrm{m}) & q=0 .\end{cases}
$$

Proof of (1), for instance, runs similarly as in Lemma 7. Thus we recall the isomorphism

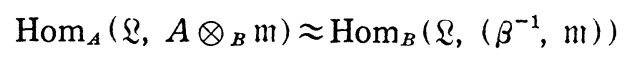

derived there from the isomorphism I, (18), i.e. $A \otimes_{B} \mathrm{~m} \approx \operatorname{Hom}_{R, \beta^{-1}}\left({ }_{B} A, \mathfrak{m}\right)$. (4) proves (1) for $q=0$. On replacing $\mathfrak{Z}$ with its $(A, B)$-projective resolution $\mathfrak{X}$ and passing to homology we obtain

$$
\operatorname{Ext}_{(A, B)}^{q}\left(\left\{, A \otimes_{B} \mathfrak{m}\right) \approx H^{q}\left(\operatorname{Hom}_{B}\left(\mathfrak{X},\left(\beta^{-1}, \mathfrak{m}\right)\right)\right)\right.
$$

and the right-hand side is 0 for $q>0$, since $\mathfrak{X} B$-splits.

Analogously, (2), (3) may be proved similarly as Lemmas $7^{\prime}, 7^{\prime \prime}$, again using I, (18).

TheOREM 18. Let $A$ be a $\beta$-Frobenius extension of $B$. If $\&$ is an A-leftmodule and if $1 \cdot \operatorname{dim}_{(A, B)} \mathfrak{Q}<\infty$, then $1 \cdot \operatorname{dim}_{(A, B)} \mathfrak{Q}=0$. The same holds with 
1. $\operatorname{dim}_{(A, B)}$ replaced either by 1 inj. $\operatorname{dim}_{(A, P)}$ or $\mathrm{l}$. w. $\operatorname{dim}_{(A, B)}$.

Proof. Let $1 \cdot \operatorname{dim}_{\left(A, U^{\prime}\right)} \mathfrak{Q}=n<\infty$. Let $\mathfrak{M}$ be an $A$-left-module such that $\operatorname{Ext}_{(A, B)}^{n, \cdot}(\mathfrak{R}, \mathfrak{N}) \neq 0$ and

$$
0 \rightarrow \mathfrak{i} \rightarrow \mathfrak{i} \rightarrow \mathfrak{M} \rightarrow 0
$$

be exact with $\hat{f} A$-left-free. Since $\operatorname{Ext}_{(A, B)}^{n+1}(\Omega, \Omega\}=0$ it follows that the induced

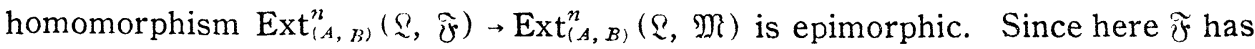
a form $\mathfrak{F}=A \otimes_{B}$ In with a $B$-left(-free) module $m, \operatorname{Ext}_{(A, B)}^{n}(\mathbb{R}, \mathfrak{F})=0$ if $n>0$, by our lemma. Hence $\operatorname{Ext}_{i A, B}^{n}(\mathfrak{Q}, \mathfrak{M})=0$ too if $n>0$. This implies that $n$ must be 0 .

The other parts of the theorem can be seen analogously.

Corollary 19. Let $A$ be a 2. Frobenius extension of $B$. Then

$$
\begin{array}{ll}
\text { 1. }(\text { resp.r }) \operatorname{gl} \operatorname{dim}(A, B)=0 & \text { or } \infty, \\
\text { w. } \operatorname{gl} \cdot \operatorname{dim}(A, B)=0 & \text { or } \infty .
\end{array}
$$

Remark. In analogy to this corollary, we could state the following corallary to Theorems $8,8^{\prime}, 8^{\prime \prime}, 9,9^{\prime}, 9^{\prime \prime}$ in $\mathrm{I}, \S 3$ :

Corollary 9 a. Let $A$ be 2.Frobenius over $B$ and assume that $B$ is $\gamma-1$ isomorphic to a direct summand of the $B$-B-module $A$. Then

$$
\begin{array}{ll}
\text { 1. (resp.r.)gl.dim } A=1 .(\text { resp.r.) gl. } \operatorname{dim} B & \text { or } \infty \\
\text { w. gl. } \operatorname{dim} A=\text { w.gl. } \operatorname{dim} B & \text { or } \infty .
\end{array}
$$

The equation $1 \cdot \operatorname{dim}_{(A, B)} \mathscr{Q}=0$ (which we have, in case $A$ is 2 .Frobenius over $B$, seen to be the case whenever $\left.1 \cdot \operatorname{dim}_{(A, B)} \mathfrak{Q}<\infty\right)$ means that $\mathfrak{Q}$ is (relatively) ( $A, B$ )-projective. It is well known (cf. Hochschild [7]) that with any $B$-leftmodule $\mathrm{m}$ the $A$-left-module $A \otimes_{B} \mathrm{~m}$ is $(A, B)$-projective and conversely any ( $A, B$ )-projective $A$-left-module $\Omega$ is a direct summand of an $A$-left-module of form $A \otimes_{B}$ lit, for example $A \otimes_{B} \Omega$. On the other hand, in case $A$ is 2 .Frobenius over $B$, Lemma $17,(1)$ shows that $A \otimes_{B} \mathfrak{m}$ is $(A, B)$-injective and hence any ( $A, B$ )-projective $A$-left-module is $(A, B)$-injective too. The converse is seen from (a well known general fact on $(A, B)$-injective modules and) Lemma 17 , (2). Thus

Theorem 20. A left-module \& over a 2.Frobenius extension $A$ of $B$ is ( $A$, $B)$-projective if and only if it is $(A, B)$-injective. 
The above mentioned fact, that an $(A, B)$-projective $A$-left-module $\&$ is a direct summand of $A \otimes \otimes_{B} \stackrel{Q}{2}$, is seen by considering the $A$-epimorphism $\rho$ of

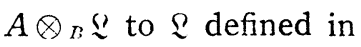

$$
\rho\left(\sum a_{i} \otimes l_{i}\right)=\sum a_{i} l_{i} \quad\left(a_{i} \in A, l_{i} \in \mathbb{Z}\right)
$$

and observing that Ker $\rho$ is a direct summand of $A \otimes_{B} \mathscr{Q}$ as a $B$-left-module (as we see by the $B$-monomorphism $l \rightarrow 1 \otimes l$ of $\stackrel{i}{i}$ into $A \otimes_{B} \&$ ). The last is the case generally, and $\mathscr{Q}$ is $(A, B)$-projective if and only if $\operatorname{Ker} \rho$ is a direct summand of the $A$-left-module $A \otimes_{B} \Omega$. Similarly the $(A, B)$-injectivity of an $A$-left-module $\mathfrak{Z}$ is reduced to the image of a certain canonical $A$-monomorphism $\mathfrak{I} \rightarrow \operatorname{Hom}_{B}(A, \mathfrak{Q})$ being a direct summand of the $A$-left-module $\operatorname{Hom}_{B}(A, \mathfrak{R})$.

In case $A$ is $\beta$-Frobenius over $B$, we have an $A$-isomorphism $A \otimes_{B}(\beta, \mathbb{Q})$ $\approx \operatorname{Hom}_{B, \beta^{-}}(A,(\beta, \Omega))=\operatorname{Hom}_{B}(A, \Omega)$, by $\mathrm{I},(18)$ (used above repeatedly). In combination with the above remark it follows that the $(A, B)$-injectivity too can then be characterized by means of a tensor product, $A \otimes_{B}(\beta, \Omega)$, and indeed by that the image of a certain canonical $A$-monomorphism $\mathbb{R} \rightarrow A \otimes_{B}(\beta, \mathfrak{Q})$ is a direct summand of $A \otimes_{B}(\beta, \Omega)$ as $A$-module.

This last we have seen without making use of Theorem 20 (If we use it. it is clear that the $(A, B)$-injectivity of $\mathbb{Z}$ is, in case $A$ is 2 .Frobenius over $B$,

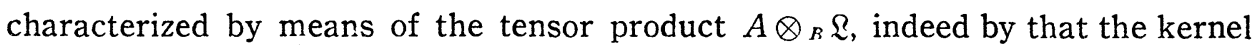
of a canonical $A$-epimorphism $A \otimes_{B} \mathfrak{Z} \rightarrow \mathfrak{Z}$ is a direct summand of $A \otimes_{B} \mathfrak{Z}$ as $A$-module.) In fact Theorem 20 may be derived directly from the above remark (and its dual) readily. For, an $(A, B)$-injective module $\Omega$ is a direct summand of $A \otimes_{B}(\beta, \Omega)$ as $A$-module, by the above remark. The last module is, being of form $A \otimes_{B} \mathrm{~m},(A, B)$-projective, and hence its direct summand $\mathfrak{Q}$ is so too. This proves one half of Th.20, and the other half is seen dually.

Now, if $A$ is further quasi-free over $B$, Theorem 20 can be made more explicit in

THEOREM 21. Let $A$ be quasi-free $\beta$-Frobenius over $B$ and let $u_{\nu}, v_{\nu}$ be as in Prop. 10. Then an A-left-module $\mathfrak{Q}$ is $(A, B)$-projective if and only if there

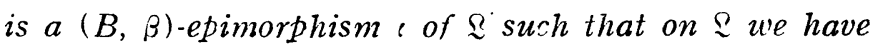

$$
\sum_{i} v_{\nu}: u_{\nu}=1
$$

The same condition is necessary and sufficient for the $(A, B)$-injectivity too. 
Proof. Suppose that $\&$ is $(A, B)$-projective, i.e. that Ker $\rho$ (which is always a direct summand of $A \otimes_{B} \&$ as $B$-module) is a direct summand of $A \otimes_{B} \&$ as $A$-module. - Then there is a direct summand $A$-submodule $\Omega_{0}$ of $A \otimes_{B} \stackrel{Q}{2}$ which is $A$-isomorphic to $\mathbb{Z}$ (in fact $A \otimes_{B} \mathfrak{Q}=\mathfrak{L}_{0} \oplus \operatorname{Ker} \rho$ ). Let $\lambda$ be the projection of $A \otimes_{B} \mathbb{Q}$ onto $\mathfrak{Q}_{n}$, with respect to a direct decomposition of $A \otimes_{B} \mathbb{Z}$ into $\Omega_{0}$ and a second $A$-submodule (which may be Ker $\rho$, but not necessarily). Let, on the other hand, $\kappa$ be the $\left(B, \beta^{-1}\right)$-endomorphism of $A \otimes_{B} \&$ defined by

$$
\kappa\left(\sum x_{i} \otimes l_{i}\right)=1 \otimes \sum\left(\beta^{-1} \pi x_{i}\right) l_{i} \quad\left(x_{i} \in A, l_{i} \in \mathfrak{Q}\right)
$$

(i.e. $\kappa=\beta^{-1} \pi \otimes 1$ ). As an operator on $A \otimes{ }_{B} \&$ we have

$$
\sum_{\nu} v_{\nu} \kappa u_{\nu}=1
$$

For, $\left(\sum_{\nu} v_{\nu} \kappa u_{\nu}\right)(x \otimes l)=\sum_{\nu} v_{\nu} \otimes\left(\beta^{-1} \pi u_{\nu} x\right) l=\sum_{\nu} v_{\nu} \beta^{-1} \pi u_{\nu} x \otimes l=$ $\sum_{\nu} v_{\nu} \beta^{-1} \pi \sum_{\mu}\left(\beta y_{\nu \mu}\right) u_{\mu} \otimes l=\sum_{i l} \sum_{\nu} v_{\nu} \beta^{-1}\left(\beta y_{\nu: L}\right) \pi u_{\mu} \otimes l=\sum_{\mu} \sum_{\nu} v_{\nu}, v_{\nu \mu} \beta^{-1} \pi u_{\mu} \otimes l=$ $\sum_{\mu} x v_{\mu} \beta^{-1} \pi u_{\mu} \otimes l=x \otimes l$

by I, (42), (41) and (40). Since $\lambda$ is $A$-homomorphic, it follows that $\sum_{\nu} v_{\nu} \lambda \kappa u$. $=\lambda \sum_{\nu} v_{\nu} \kappa u_{\nu}=\lambda$ and induces the identity map on $\Omega_{0}$. On denoting by 6 the $(B, \beta)$-endomorphism of $\mathfrak{Q}$ which corresponds to $\lambda \kappa$ on $\mathfrak{S}_{0}$, by an $A$-isomorphism of $\mathfrak{L}$ and $\mathfrak{L}_{0}$, we odtain $(6)$.

Now, let $(6)$ be the case. Let $\mathfrak{M}$ be an $A$-left-module having $\mathfrak{Q}$ as an $A$ submodule such that $\mathfrak{M}$ is the direct sum of $\mathfrak{Q}$ and a $B$-submodule. Let $\lambda_{1}$ be the projection of $\mathfrak{M}$ onto $\mathfrak{Z}$, with respect to this direct decomposition of $\mathfrak{M}$, and let $\lambda_{l}$ be the map $\sum_{v} v_{v} \iota \lambda_{1} u_{\nu}$ on $\mathfrak{M} . \quad \lambda_{2}$ maps $\mathfrak{M} A$-homomorphically into $:$ as the relations $\mathrm{I},(41),(42)$ and the $(B, \beta)$-homomorphic feature of : show. Further, $\lambda_{2}$ induces the identity map on $\mathbb{Q}$, as the relation (6) entails. Thus $\mathfrak{M}=\mathfrak{Q} \oplus \operatorname{Ker} \lambda_{2}$ is a direct $A$-decomposition and this shows that $\mathfrak{Z}$ is $(A, B)$. injective.

Lastly, assume that $\mathbb{Q}$ is $(A, B)$-injective. Then $\mathscr{Q}$ is $(A, B)$-projective, by Theorem 20 .

This closes a circuit and proves our Theorem 21. (The last step in the above circuit, concluding the $(A, B)$-projectivity from the $(A, B)$-injectivity, is seen also by means of $A \otimes_{B}(\beta, \Omega)$, as was remarked above. An $A$-monomorphism of $\&$ into $A \otimes_{3}(\beta, \&)$, used in that argument, may be given, in case $A$ is quasi-free $\beta$-Frobenius over $B$, by 


$$
\sigma: l \rightarrow \sum_{\nu} v_{\nu} \otimes(u, l)^{\prime} \in A \otimes_{n}(\beta, 尺) \quad(l \in \unrhd)
$$

where $l^{\prime}$ denotes the element of $(\beta, \Omega)$ corresponding to an element $l$ of $\&$. For, the $A$-homomorphic feature of $\sigma$ may be seen in

$$
\begin{aligned}
\sigma x l & =\sum_{\nu} v_{\nu} \otimes\left(u_{\nu} x l\right)^{\prime}=\sum_{\mu, \nu} v_{\nu} \otimes\left(\left(\beta y_{\nu \mu}\right) u_{\nu}\right)^{\prime} \\
& =\sum_{\mu, \nu} v_{\nu} \otimes y_{\nu \mu}\left(u_{\nu} l\right)^{\prime}=\sum_{\mu, \nu} v_{\nu} y_{\nu \mu} \otimes\left(u_{\nu} l\right)^{\prime} \\
& =\sum_{\mu}^{1} x v_{\mu} \otimes\left(u_{\nu} l\right)^{\prime}=x \sigma l,
\end{aligned}
$$

$y_{\nu \mu}$ being as in I, (41), (42). That $\operatorname{Im} \sigma$ is a direct summand of $A \otimes_{B}(\beta, \&)$ as mere $B$-module, is seen by means of the $B$-homomorphism $\sigma_{1}$ of $A \otimes_{B}(\beta, \Omega)$ to $\&$ defined by $\sigma_{1}\left(\sum a_{i} \otimes l_{i}^{\prime}\right)=\sum\left(\pi a_{i}\right) l_{i}$ (observe that $\pi$ is $(B, 1)-(B, \beta)$. homomorphic) and by observing that $\sigma_{1} \sigma l=\sigma_{1}\left(\sum v_{\nu} \otimes\left(u_{\nu} l\right)^{\prime}\right)=\left(\pi v_{\downarrow}\right) u_{\downarrow} l=l$ (by I, (40)), i.e. that $\sigma_{1} \sigma$ is identity on 2 . This explicit way and the similarly explicit construction in the proof of Theorem 21 form the generalization of the Maschke-Ikeda-Kasch [10] argument to our present case of a quasi-free 2.Frobenius extension.)

\section{Endomorphism ring.}

With a ring $A$ having a subring $B$, consider the $B$-rightendomorphism ring F of $A$; the elements of $F$ are considered as left-operators on $A$. The ring $A_{l}$ of left-multiplications of elements of $A$, on $A$, is a subring; with a subset $S$ of $A$ we denote by $S_{l}$ the set of left-multiplications of elements of $S$ on $A$.

We prove the following refinement of Kasch [10], Satz 5 and NakayamaTsuzuku [13], Theorem :

TheOREm 22. Let $A$ be an extension ring of a ring $B$. Let $\beta$ be an automorphism of $B$ which can be extended to an automorphism, say $\alpha$, of $A$, and denote by $\alpha^{\prime}$ the automorphism of the left-multiplication ring $A_{l}$ of $A$ corresponding to $\alpha^{-1}$. If $A$ is quasi-free $\beta$-Frobenius over $B$, then the ring (F) of $B$-rightendomorphisms of $A$ is quasi-free $\alpha^{\prime}$-Frobenius over $A_{l}$. Conversely, if (5) is $\alpha^{\prime}$. Frobenius over $A_{l}$, then $A$ is quasi-free $\beta$-Frobenius over $B$, provided that $A$ is finitely generated B-left-quasi-free.

Proof. We first prove the second half of the theorem. Thus, let

$$
A=v_{1} B+\cdots+v_{n} B
$$


be, as in $I,(33)$, a direct decomposition of $A$ into $B$ right-submodules $v_{\vee} B$ such that $v_{\nu} B \approx e_{\nu} B$ ( $B$-right), $v_{\nu} e_{\nu}=v_{\nu}$, where $\epsilon_{\nu}$ are idempotents in $B$. Let $E_{\nu}$ be, for each $\nu=1, \ldots, n$, the $B$-right-endomorphism of $A$ such that

$$
E_{\nu} v_{\nu}=e_{\nu}(\in B \subset A), \quad E_{\nu} v_{\mu}=0 \quad(\mu \neq \nu) .
$$

As $v_{\nu}$ is mapped by any $B$.right-endomorphism of $A$ onto an element in $A e_{\nu}$, we readily see the direct decomposition

$$
E=\left(A e_{1}\right)_{l} E_{1}+\cdots+\left(A e_{n}\right)_{l} E_{n} .
$$

By the assumption that $\&$ is $\alpha^{\prime}$-Frobenius over $A_{l}$ there exists, according to Prop. 4 , an $\left(A_{l}, 1\right)-\left(A_{l}, \alpha^{\prime}\right)$-homomorphism $A$ of $\&$ to $A_{l}$ such that $A \mathfrak{E} \varphi=0$ $(\varphi \in(\xi)$ implies $\varphi=0$.

Set $w_{\nu l}=A E_{\downarrow}\left(w_{\downarrow} \in A\right.$, in fact $\left.\in e_{\downarrow} A\right)$. We contend that a relation

$$
y_{1 l} w_{1 l}+\cdots+y_{n l} w_{n l}=0 \quad\left(y_{\nu} \in B e_{\nu}\right)
$$

can hold only when $y_{1}=\cdots=y_{n}=0$. To prove this, set

$$
x_{\nu}=v_{1} y_{\nu}+\cdots+v_{n} y_{\nu}=\left(v_{1}+\cdots+v_{n}\right) y_{\nu} \in A e_{\nu}
$$

and consider the elements

$$
X_{\mu}=E_{\mu}\left(x_{1 l} E_{1}+\cdots+x_{n l} E_{n}\right)
$$

of $\varepsilon$. For each $\mu, \nu$ and $b \in B$ we have

$$
\begin{aligned}
X_{\mu} v_{\nu} b & =E_{\mu}\left(x_{1 l} E_{1}+\cdots+x_{n l} E_{n}\right) v_{\nu} b \\
& =E_{\mu} x_{\nu} e_{\nu} b=E_{\iota} x_{\nu} b=E_{\mu}\left(v_{1}+\cdots v_{n}\right) y_{\nu} b=e_{\mu} y_{\nu} b .
\end{aligned}
$$

Since this is the case for every $\nu=1, \ldots, n$, we see

$$
X_{\mu}=\left(e_{\mu} y_{1}\right)_{l} E_{1}+\cdots+\left(e_{\mu} y_{n}\right)_{l} E_{n}=e_{\mu l}\left(y_{1 l} E_{1}+\cdots+y_{n l} E_{n}\right) .
$$

Hence

$$
\Lambda X_{\mu}=\Lambda\left(y_{1 l} E_{1}+\cdots+y_{n l} E_{n}\right) \boldsymbol{e}_{\mu l}=\left(y_{1 l} w_{1 !}+\cdots+y_{1 !} w_{1 l}\right) \alpha e_{\mu l}
$$

and this is 0 by the assumed relation (12). Then $\Lambda x_{l} X_{\mu}=x_{l} \Lambda X_{i \mu}=0$ for any $x \in A$, i.e.

$$
\Lambda x_{l} E_{\mu}\left(x_{1 l} E_{1}+\cdots+x_{n l} E_{n}\right)=0
$$

for any $x \in A$. As this holds for every $\mu=1, \ldots, n$, we have, in view of (11),

$$
.1 \mathfrak{E}\left(x_{1 l} E_{1}+\cdots+x_{n l} E_{l}\right)=0
$$

and hence 


$$
x_{1 l} E_{1}+\cdots+x_{n l} E_{n}=0
$$

As $x_{\nu} \in A e_{\nu}$, we have then $x_{\nu}=0$, for each $\nu=1, \ldots, n$. Then $y_{\nu}=0, \nu=1$, $\therefore, n$, as was asserted.

So we obtain a direct sum submodule

$$
\left(B e_{1}\right)_{l} w_{1 l}+\cdots+\left(B e_{n}\right)_{l} w_{n l}
$$

of $A_{l}$, where each summand $\left(B e_{\nu}\right)_{l} w_{\nu l}$ is $B_{l}$-left-isomorphic to $B_{l} e_{v l}$; remember that $B_{l}$ is the set of the left-multiplications of the elements of $B$ onto $A$ (not onto $B$ ).

Next we prove that the sum

$$
B_{l} E_{1}+\cdots+B_{l} E_{n}
$$

is a right-ideal in $\left(5=A_{l} E_{1}+\cdots+A_{l} E_{n}((11))\right.$. Namely, with $x=v_{1} y_{1}+\cdots$ $+v_{n} y_{n} \in A\left(y_{\nu} \in e_{\nu} B\right)$ and $y \in B$ we have

$$
\begin{aligned}
y_{l} E_{\mathrm{\kappa}} x_{l} E_{\nu} v_{\mu} b & =\delta_{\mu \nu} y_{l} E_{\kappa} x_{l} e_{\nu} b=\delta_{\mu \nu} y_{l} E_{\kappa}\left(v_{1} y_{1}+\cdots+v_{n} y_{n}\right) e_{\nu} b \\
& =\delta_{\mu \nu} y_{l} e_{\kappa} y_{\kappa} e_{\nu} b=\delta_{\mu \nu} y_{.} y_{\kappa} e_{\nu} b
\end{aligned}
$$

for any $b \in B$ and $\kappa$, which means

$$
y_{l} E_{\kappa} x_{l} E_{\nu}=\left(y y_{\kappa}\right)_{l} E_{\nu} \in B_{l} E_{\nu} .
$$

This shows $B_{l} E_{\kappa} E \subseteq B_{l} E_{1}+\cdots+B_{l} E_{n}$, for any $\kappa$, and therefore (13) is a right-ideal of $(5$.

As $A$ is $\left(A_{l}, \alpha^{\prime}\right)$-right-homomorphic, it follows that $A\left(B_{l} E_{1}+\cdots+B_{l} E_{n}\right)$ $=B_{l} w_{1 l}+\cdots+B_{l} w_{n l}$ is a right-ideal of $A_{l}$ and indeed an $B_{l}$-left, $A_{l}$-rightsubmodule of $A_{l}$.

Another consequence of the right-ideal property of $B_{l} E_{1}+\cdots+B_{l} E_{n}$ is, as we see by virtue of Prop. 4 , ii) (with $A, B, \beta$ replaced by $\left(F A_{l}, \alpha^{\prime}\right.$ ), that by any $\left(A_{l}, \alpha^{\prime-1}\right)$-left-homomorphism of $\&$ into $A_{l}$ every element in $B_{l} E_{1}+$ $\cdots+B_{l} E_{n}$ is mapped into $\alpha^{\prime-1} A\left(B_{l} E_{1}+\cdots+B_{l} E_{n}\right)=\alpha^{\prime-1}\left(B_{l} w_{1 l}+\cdots+\right.$ $\left.B_{l} w_{1 l}\right)$. But, on the other hand, we see by (11) the existence of an $\left(A_{l}, \alpha^{1-1}\right)$. left-homomorphism of (; into $A_{l}$ mapping $E_{\imath}$ onto an arbitrarily given element of $\alpha^{\prime^{-1}}\left(A e_{\nu}\right)_{l}$. Hence we have $\alpha^{1^{-1}}\left(B_{l} w_{1 l}+\cdots+B_{l} w_{n l}\right) \supseteq \alpha^{\prime-1}\left(A e_{\nu}\right)_{l}$, or equivalently,

$$
B_{l} w_{1 l}+\cdots+B_{l} w_{n l} \supseteq\left(A e_{\nu}\right)_{l} .
$$

Since the left-hand side is a right-ideal of $A_{l}$, as observed above, we have 
$B_{l} w_{1 l}+\cdots+B_{i} v_{1 l} \supseteq\left(\Lambda e^{\prime}, A\right)_{l}$, or, what is the same

$$
B w_{1}+\cdots+B w_{n} \supseteq A \ell, A .
$$

As this holds for every $\nu^{\prime}=1, \ldots, n$ and as $A e_{1} A+\cdots+A e_{n} A=A$ by (9) (and $v_{\nu} e_{s}=v_{\nu}$ ), we obtain

$$
A=B w_{1}+\cdots+B w_{n}
$$

where, as we proved above with respect to $A_{l}$ in stead of $A$ itself, the sum is direct and each $B w_{\nu}$ is $B$-left-isomorphic to $B e_{2}$.

Now we wish to show that the elements $u_{\nu}=\alpha w_{\nu}, v_{\nu}$ are in the dual relationship as in Prop. 11. Thus, if, with an element $x$ of $A$, we have $I$, (41), i.e.

$$
x v_{\nu}=\sum_{\mu=1}^{n} v_{\mu} y_{\mu \nu} \quad\left(y_{\mu \nu} \in e_{\mu \nu} B e_{\nu}\right)
$$

then we have $E_{i\llcorner} x_{!}=\sum_{\nu} y_{i, \nu l} E_{\nu}$ as is seen from

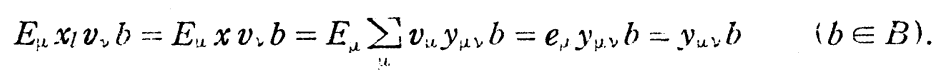

Applying $A$ we have $2 w_{\mu l} \alpha^{\prime} x_{l}=\sum_{i} y_{\mu \nu l} w_{\nu l}$ or $\left(\alpha^{\prime-1} w_{u l}\right) x_{l}=\sum_{i}\left(\alpha^{\prime-1} v_{\mu \nu l}\right) \alpha^{\prime-1} w_{\nu l}$. Setting $u_{\mu}=\alpha w_{j}(\mu=1, \ldots, n)$ we obtain

$$
\boldsymbol{u}_{\mu} x=\sum_{\nu=1}^{n}\left(\beta y_{\mu \nu}\right) \boldsymbol{u}_{\nu}
$$

i.e. I, (42); observe that $\beta$ is the restriction to $B$ of the automorphism $\alpha$ of $A$ which in turn corresponds to the automorphism $\alpha^{1^{-1}}$ of $A_{l}$. Here $\left(\beta e_{2}\right) u$. $=\left(\alpha e_{\nu}\right) u_{\nu}=u_{\nu}$ and $B u_{v}$, is $B$-left-isomorphic to $B \beta e_{v}$, since $e_{\nu} w_{\nu}=u_{\nu}$, and $B u^{\prime}$, is $B$-left-isomorphic to $B e_{2}$. Further we have

$$
A=B u_{1}+\cdots+B u_{n}
$$

and the sum is direct, as again the application of $\alpha^{-1}$ reduces the case to that of $w_{\nu}$.

So $A$ is $\beta$-Frobenius over $B$, and the second half of our theorem is proved. The verification of the first half is rather straight-forward. Thus, assume that $A$ is quasi-free $\beta$-Frobenius over $B$. We have $\mathrm{I},(33),(3 \overline{5})$, in notations described in Prop 10, and I, (41), (42) entailing each other as in Prop. 11. Writing I, (33) in the form (9) (as Prop. 10 tells) and defining $E_{2} \in(5$, as in (10), we obtain the direct decomposition (11). By the assumption of our theorem $\beta$ is 
extended to an automorphism $\alpha$ of $A$, and we denote also by $\alpha$ the automorphism of $A_{l}$ naturally induced by it. To each element $X$ of $(5$ we associate an element $\varphi_{X}$ of $\operatorname{Hom}_{A^{4}, \alpha}\left(A_{A_{l}}\left(\tilde{E},{ }_{A_{l}} A_{l}\right)\right.$ defined by

$$
\varphi_{X} Y=\sum_{\nu=1}^{n}\left(\alpha Y X v_{\nu}\right)_{l u, l} \quad(Y \in(\xi) .
$$

with our "dual basic elements" $u_{\nu}, v_{\nu}$. Then the map $X \rightarrow \varphi_{X}$ is an $A_{l}$-right homomorphism as we see from the relations $I,(41),(42)$. The map is evidently (5.-left-homomorphic too.

We wish to show that the map is an isomorphism. Let, for this purpose, $X=x_{1 l} E_{1}+\cdots+x_{n l} E_{n}\left(x_{2} \in A e_{2}\right)$ be a non-zero element of $\mathfrak{F}$. There exists an index, say $\nu_{0}$, with $x_{\nu_{0}} \neq 0$. If we write $x_{\nu}=\sum_{\mu=1}^{n} v_{\mu} z_{\mu \nu}\left(z_{\mu \nu} \in e_{\mu} B\right)$ for each $\nu$, then $z_{\mu_{0} \nu_{0}} \neq 0$ for some $\mu_{3}$. We have

$$
\begin{aligned}
\varphi_{X} E_{\nu_{0}} & =\sum_{\nu=1}^{n}\left(\alpha E_{\nu_{0}} X v_{\nu}\right)_{l} u_{\nu l}=\sum_{\nu=1}^{n}\left(\alpha E_{\nu_{0}} x_{\nu}\right)_{l} u_{\nu l}=\sum_{\nu=1}^{n}\left(\alpha z_{\nu_{0} \nu}\right) \boldsymbol{u}_{\nu l} \\
& =\left(\sum_{\nu=1}^{n}\left(\beta z_{\nu_{0} \nu}\right) u \nu\right)_{l} \neq 0 .
\end{aligned}
$$

Hence $\varphi_{X} \neq 0$, and this proves that our map is monomorphic.

Further, for arbitrarily given elements $b_{\nu} \in B e_{\nu}(\nu=1, \ldots, n)$, set

$$
X=\sum_{\nu=1}^{n}\left(v_{1} b_{\nu}\right), E_{\nu}
$$

Then

$$
\begin{aligned}
\varphi_{X} E_{\mu}= & \sum_{\nu=1}^{n}\left(\alpha E_{\mu} X v_{\nu}\right)_{l} u_{\nu l}=\sum_{\nu=1}^{n}\left(\alpha E_{\mu} v_{1} b_{\nu}\right)_{l} u_{\nu l}= \\
& \left\{\begin{array}{l}
\sum_{\nu=1}^{n}\left(\alpha e_{1} b_{\nu}\right)_{l} u_{\nu l}=\left(\left(\beta e_{1}\right) \sum_{\nu=1}^{n}\left(\beta b_{\nu}\right) u_{\nu}\right)_{l} \quad \text { for } \mu=1, \\
0 \quad \text { for } \mu \neq 1
\end{array}\right.
\end{aligned}
$$

Similarly, for each $\nu$ and for every element $x$ of $A$ there exists an $X$ in $(5$ such that $\varphi_{X} E_{\mu}=\left(\left(\beta e_{2}\right) x\right)_{l}$ or 0 according as $\mu=\nu$ or $\mu \neq \nu$. This proves that our map $X \rightarrow \varphi_{X}$ is epimorphic too.

Thus $\mathfrak{F}_{A_{l}} \approx \operatorname{Hom}_{A_{l, \alpha}}\left({ }_{A_{l}}\left(\mathfrak{F},{ }_{A l} A_{l}\right)\right.$ and $\left(E\right.$ is (quasi-free) $\alpha^{\prime}$-Frobenius over $A_{l}$, where $\alpha^{\prime}$ denotes, as in our theorem, the inverse of the automorphism $\alpha$ of $A_{l}$. The first half of our theorem is thus proved too.

Remark. The necessity of the rather strong assumption on the extendability (to $\alpha$ ) of the automorphism $\beta$, in our theorem, seems to come from 
the midway feature of our definition of 2.Frobenius extensions mentioned already in a Remark at the end of $I, \S 1$. We intends to come to this point in a subsequent .paper.

Remark. Kasch [10] uses his Satz j (which we have generalized to our Theorem 22 ) to prove that if $(B$ is a semiregular automorphism group (in the sense of [18]) of a simple ring with minimum condition $A$ (which means that the totality of inner automorphisms of $A$ contained in $\mathbb{f}$ forms an (invariant) subgroup $\mathbb{G}_{0}$ of finite index in $(\vec{S}$ and is indeed the set of inner automorphisms of $A$ induced by (all) the regular elements of a semisimple subring $T$ of $A$ having finite rank over the center $C$ of $A$ ) then $A$ is a Frobenius extension of the fixed subring $B$ of 8 . As Kasch [10] briefly remarks, the same holds with a complete automorphism group of (a simple ring with minimum condition) $A$ whose subgroup of inner automorphisms is of finite index and is induced by (all) the regular elements of a subring $T$ of $A$ Frobenius over $C$, the center of $A$, provided that the product of $T_{r}$ and $A_{l}$ is a tensor product over $C_{r}\left(=C_{l}\right)$ and $A$ is projective with respective to the endomorphism ring of $A$ generated by, (S) and $A_{l}$; Curtis [14] discusses when this last is the case.

One might try to make a further generalization by replacing here "Frobenius". with our "2.Frobenius". But this would be rather meaningless. For, firstly, since $C$ is a field, every 2.Frobenius extension $T$ of $C$ is free and, moreover, since $C$ should naturally be in the center of $T, T$ must be 1 .Frobenius. Namely, if $A$ is a $\beta$-Frobenius extension of $a$ ring $B$ and if $B$ is contained in the center of $A$, then necessarily $\beta=1$. For, there is, by Prop. 4, $a(B, 1)$ $(B, \beta)$-homomorphism $\pi$ of $A$ into $B$ such that $\pi a A=0 \quad(a \in A)$ entails $a=0$. So, with $b \in B, x \in A$, we have, since $b, \beta b$ lie in the center of $A, \pi b x=\pi x b$ $=(\pi x) \beta b=(\beta b) \pi x=\pi(\beta b) x, \pi(b-\beta b) x=0$. As $x$ is arbitrary in $A, \pi(b-\beta b) A$ $=0$ and this entails $b-\beta b=0$. It follows that $\beta=1$.

(If we should consider a Galois theory of non-simple, and perhaps nonsemisimple, rings, then it would probably be useful to consider "non-free 1.Frobenius" case. However, as the developement of Galois theory for such general rings seems still to be at an unsatisfactory stage (except with the outer Galois theory), we shall not go into such a consideration here).

In this context we want to remark, however, that the field property makes no hindrance for having a non-1.Frobenius 2.Frobenius extension. As the 
examples given in I, $\$ 2$ were with respect to non-field subrings, we thus give here

Example 3. Let $K$ be a field having an involutive automorphism $(\neq 1)$ $\vec{\xi} \rightarrow \vec{\xi}$, and let $A$ be the ring of all matrices of form

$$
x=\left(\begin{array}{cc}
\xi & 0 \\
\zeta & \bar{\xi}
\end{array}\right) \quad(\xi, \zeta \in K)
$$

in $K$. Let $B$ be the subring of $A$ consisting of all $x$ with $\zeta=0$. Clearly $B$ is a field isomorphic to $K$, and has an (involutive) automorphism $\beta$ mapping $\left(\begin{array}{ll}\xi & 0 \\ 0 & \bar{\xi}\end{array}\right)$ to $\left(\begin{array}{ll}\hat{5} & 0 \\ 0 & \xi\end{array}\right)$. Now, set

$$
\pi x=\left(\begin{array}{ll}
\zeta & 0 \\
0 & \zeta
\end{array}\right) \quad(\in B) .
$$

If $x_{1}=\left(\begin{array}{cc}\xi_{1} & 0 \\ \zeta & \bar{\xi}\end{array}\right)$, then $x x_{1}=\left(\begin{array}{cc}\xi \xi_{1} & 0 \\ \zeta \xi_{1}+\bar{\xi} \zeta_{1} & \bar{\xi} \bar{\xi}_{1}\end{array}\right)$ and hence

$$
\pi x x_{1}=\left(\begin{array}{cc}
\bar{\zeta} \bar{\xi}_{1}+\hat{\zeta} \bar{\zeta}_{1} & 0 \\
0 & \zeta \xi_{1}+\bar{\xi} \zeta_{1}
\end{array}\right) .
$$

From this relation we see readily that $\pi$ is a $(B, 1)-(B, \beta)$-homomorphism of $A$ to $B$. From the same relation we see also immediately that the condition $i_{r}$ ) of Prop. 4 holds. On the other hand, 1 and $u=\left(\begin{array}{ll}0 & 0 \\ 1 & 0\end{array}\right)$ form a free $B$-rightbasis of $A$. Hence $\operatorname{Hom}_{B, \beta}\left(A_{B}, B_{B}\right)$ is a free $B$-left-module of rank 2. Hence we have a $B$-A-monomorphism $a \rightarrow \varphi_{a}=\pi^{a}$ of ${ }_{B} A_{A}$ into $\operatorname{Hom}_{B, \beta}\left(A_{E}, B_{B}\right)$. Since $(1, u)$ is also a free $B$-left-basis of $A$, the map $a \rightarrow \varphi_{a}=\pi^{a}$ is epimorphic too. $A$ is thus a 2.Frobenius extension of the field $B$ with respect to $\beta$. (In fact, $(1, u)$ and $(u, 1)$ are $B$-right and $B$-left free bases of $A$ dual in the sense of Prop. 10, 12). Since the automorphism $\beta$ of $B$ can not be induced by a transformation by a regular element in $A, A$ is not 1.Frobenius over $B$. (This example is closely related to Example 2 in I, $\S 2$. Indeed, if we consider our $A$ as an algebra over the fixed subfield $L$ of the automorphism $\xi \rightarrow \xi$ in $K$ and pass to the tensor product $A \otimes_{L} K$, then we come back to the case of Example 2).

\section{\$. Annihilators of ideals.}

Let $A$ be $\beta$-Frobenius over $B$. By means of the $(B, 1)-(B, \beta)$-homomorphism $\pi=\emptyset 1$, as in Prop. 4, we defined in $\$ 2$ 


$$
\begin{aligned}
& R_{\pi} X=\{x \in A \mid \pi X x=0\} \\
& L_{\pi} X=\{x \in A \mid \pi x X=0\}
\end{aligned}
$$

for a subset $X$ of $A$.

Lemma 24. $L_{\pi} A X$ is a right ideal of $A$ and is indeed the largest right ideal of $A$ contained in $L_{\pi} X$.

Proof. As $A X$ is a left ideal, we see readily that $L_{\pi} A X$ is a right ideal in $A$. Clearly $L_{\pi} A X \subseteq L_{-} X$. Further, if $\mathrm{r}$ is a right ideal of $A$ contained in $L_{\pi} X$, then

$$
\pi \mathrm{r} A X=\pi \mathrm{r} X=0
$$

whence $\mathfrak{r} \subseteq L_{\pi} A X$. (The lemma will be made use of in the next section).

Lemma 25. $L_{\pi} X A$ is a left ideal of $A$ and is indeed the largest left ideal of $A$ contained in $L_{\pi} X$. Moreover, $L_{\pi} X A$ conicides with the left annihilator (i.e. the set of all left annihilators, to be more precise) lXA of XA in $A$.

Proof. Denote the right ideal $X A$ by r. As $(l \mathfrak{r}) \mathfrak{r}=0$ we have clearly $l \mathfrak{r} \subseteq L_{\pi} \mathfrak{r}$. Conversely, from $\pi\left(L_{\pi} \mathfrak{r}\right) \mathfrak{r}\left(=\pi\left(L_{\pi} \mathfrak{r}\right) \mathfrak{r} A\right)=0$ we obtain $\left(L_{\pi} \mathfrak{r}\right) \mathfrak{r}=0$ (i.e. $\left.l \mathfrak{r} \supseteq L_{\pi} \mathfrak{r}\right)$ by virtue of Prop. $4, \mathfrak{i}_{r}$ ). Thus $L_{\pi} \mathfrak{r}=l \mathfrak{r}$, proving the second half of the lemma. Further, clearly $L_{\pi} \mathfrak{r}=L_{\pi} X A \subseteq L_{*} X$. If $\mathfrak{l}$ is a left ideal of $A$ contained in $L_{\pi} X$, then $\pi l X=0, \pi A l X=0$, whence $\left\{X=0\right.$, by Prop. $\left.4, i_{l}\right)$, and therefore, $\mathfrak{l} X A=0, \pi \mathfrak{l} X A=0, \mathfrak{l} \subseteq L_{\pi} X A$. This shows that $L_{\pi} X A=L_{\pi} \mathfrak{r}$ is the largest left ideal contained in $L_{-} X$. Now, we call right $d$-ideals those right ideals of $A$ which are direct $B$-right-summands of $A$; they are similar to, but somewhat more general than, what are called right $v$-ideals in Kasch [10]. Left $d$-ideals are defined similarly.

Theorem 26. Let $A$ be a 2.Frobenius extension of $B$. For every right $d$ ideal $\mathfrak{x}$ of $A$ the left annihilator $l \mathfrak{r}$ is a left d-ideal of $A$ and its right annihilator is $r$, that is,

$$
r l r=r .
$$

(Similarly

$$
\operatorname{lrl}=1
$$

for every left d-ideal 1 of $A)$. 
Proof. This follows immediately from Prop. 6 and Lemma 25 (and its right-left symmetry).

Assume now that $B$ is semi-primary. For a finitely generated projective (whence quasi-free) $B$-right (resp. left) module $\mathfrak{R}$ (resp. $\mathfrak{Q}$ ) we denote by $(\mathfrak{R}$ : $B)_{r}$ (resp. $\left(\{: B)_{l}\right)$ the number of components in a direct decomposition of $\Re$ (resp. $\&$ ) into directly indecomposable $B$-right (resp. left) modules. We have $(\Re: B)_{r}=\left(\operatorname{Hom}_{B, \beta}\left(\Re_{B}, B\right): B\right)_{l}$ for any automorphism $\beta$ of $B$. In particular, we have $(A: B)_{r}=(A: B)_{l}$ for a (necessarily quasi-free) 2.Frobenius algebra $A$, and this common value is denoted by $(A: B)$.

Theorem 27. Let $B$ be semi-primary and $A$ be 2.Frobenius over $B$. For every right d-ideal $\mathfrak{r}$ of $A$ we have

$$
(\mathfrak{r}: B)_{r}+(\mathfrak{l n}: B)_{l}=(A: B) .
$$

Proof. Let, generally, $A=\Re \oplus \Re^{\prime}$ be a direct $B$-right decomposition of $A$. Decomposing $\mathfrak{R}, \mathfrak{R}^{\prime}$ further into directly indecomposable $B$-right modules and applying Prop. 6 to the thus obtained direct decomposition of $A$, we readily find

$$
(\Re: B)_{r}+\left(L_{\pi} \Re: B\right)_{l}=(A: B) .
$$

If in particular $\Re=\mathfrak{r}$ is a right $d$-ideal of $A$, we obtain (21), by virtue of Lemma 25.

Theorem 28. Let $B$ be semi-primary and $A$ be 2.Frobenius over $B$. For every element $a$ in $A$ such that $a A$ is a right d-ideal of $A$, the left ideal $A a$ of $A$ is $B$-left projective and we have

$$
(A a: B)_{l}=(a A: B)_{r} .
$$

Proof. We have $A=a A \oplus \mathfrak{R}^{\prime}$ with a $B$-right module $\Re^{\prime}$ and hence, by Prop. 6, $A=L_{\pi} a A \oplus L_{\pi} \mathfrak{K}^{\prime}=l a A \otimes L_{\pi} \mathfrak{R}^{\prime}$. But $A / l a A \approx A a$ ( $A$-left). Hence $A a \approx L_{\pi} \mathfrak{H}^{\prime}$ $(B$-left $)$. Thus $A a$ is $B$-left projective and $(A a: B)_{l}=\left(L_{\pi} \Re \Re^{\prime}: B\right)_{l}=(a A: B)_{r}$ proving (22).

In Theorem $28 a A$ is contended merely to be $B$-left-projective. If $B$ is an $S$-ring (cf. I, $\S 5$ ), it is a left $d$-ideal. Thus

Proposition 29. Let $B$ be an S-ring and $A$ be 2.Frobenius over $B$. Every $B$-right projective right ideal of $A$ is a right d-ideal.

Proof. By Prop. 15. 


\section{§. Residue-rings.}

With a (two-sided) ideal $z$ of an extension $A$ of $B$ satisfying $z \cap B=0$, the residue-ring $A / \mathrm{z}$ can naturally be looked upon as an extension of $B$.

Proposition 30. Let be $\beta$-Frobenius over $B$, and $z$ be a (two-sided) ideal of $A$ which is a left d-ideal and'satisfies $z \cap B=0$. If there is an element $a$ in $A$ such that

a) $R_{\pi} \mathrm{z}=A a=a A$, and

b) $\pi(x y a-x a y)=0 \quad$ for any $x \in A, y \in B$,

where $\pi=\emptyset 1$ as in Prop. 4, then the residue-ring $A / z$ is $\beta$-Frobenius over $B$.

Proof. Consider the map $\pi^{\prime}: x \rightarrow \pi x a$ of $A$ into $B$. As $\pi$ is $(B, 1)-(B, \beta)$. homomorphic and $\pi(x y a-x a y)=0$ for any $x \in A, y \in B$, we see that $\pi^{\prime}$ is also $(B, 1)-(B, \beta)$-homomorphic. Since $z=L_{\pi} R_{\pi} z=L_{\pi} a A \subseteq L_{\pi} a$, the kernel of $\pi^{\prime}, \pi^{\prime}$ induces a $(B, 1)-(B, \beta)$-homomorphism of $A^{\prime}=A / z$ into $B$, which we denote also by $\pi^{\prime}$. The kernel of the last is $L_{\pi} a / \delta$ and this contains no nonzero left-ideal in $A^{\prime}$, since $z\left(=L_{\pi} R_{\pi} \gamma\right)=L_{\pi} a A$ is, by Lemma 25 , the largest left-ideal of $A$ contained in $L_{\pi} a$. Thus the map $\pi^{\prime}$ of $A^{\prime}$ into $B$ has the property $\mathrm{i}_{l}$ ) of Prop. 4 for $A^{\prime}$ instead of $A$. For every $\psi^{\prime}$ in $\operatorname{Hom}_{B, \beta^{-1}}\left({ }_{B} A^{\prime},{ }_{B} B\right)$ there is, by Prop. 4, $\left.\mathrm{ii}_{l}\right)$, an element $a_{1}$ in $A$ such that $\psi^{\prime}(x \bmod z)=\beta^{-1} \pi x a_{1}$. We have $\beta^{-1} \pi x a_{1}=0$ for every $x \in \xi$, and therefore $a_{1} \in R_{\pi} \hat{z}=A a$. Set thus $a_{1}=a_{2} a, a_{2} \in A$. Then $\psi^{\prime}(x \bmod z)=\beta^{-1} \pi x a_{2} a=\beta^{-1} \pi^{\prime} x a_{2}$ and this means that the map $\pi^{\prime}$ of $A^{\prime}$ into $B$ satisfies Prop. 4, $\mathrm{ii}_{l}$ ) for $A^{\prime}$ too, besides $\mathrm{i}_{l}$ ). Further, since $z$ is a left $d$-ideal, $A^{\prime}=A / z$ is evidently (finite generated) $B$-left projective. Hence $A^{\prime}$ is $\beta$-Frobenius over $B$ by Prop. 4 .

Proposition 31. Let $A$ be a $\beta$-Frobenius extension of an S-ring $B$, and 3 be $a$ (two-sided) ideal of $A$ which is a left (or right) d-ideal and satisfies $₹ \cap B=0$. If there is an element $a$ in $A$ such that

$\left.\mathrm{a}^{\prime}\right) \quad z=L_{\pi} A a=L_{\pi} a A$ and $\left.\mathrm{b}\right)$ of Prop. 30 hold, where $\pi=\Phi 1$ as in Prop. 4, then the residue-ring $A / z$ is $\beta$-Frobenius over $B$.

Proof. As in our proof to the preceding proposition, we see that the map $\pi^{\prime}: x \rightarrow \pi x a$ of $A$ into $B$ is $(B, 1)-(B, \beta)$-homomorphic and its kernel is $L_{\pi} a$ and contains $z\left(=L_{\pi} a A \subseteq L_{\pi} a\right)$. By $z=L_{\pi} A a=L_{\pi} a A$ and by Lemmas 24, 25, $z$ is both the largest right-ideal and the largest left-ideal of $A$ contained in the 
kernel $L_{\pi} a$ of $\pi^{\prime}$. Hence the kernel $L_{\pi} a / z$ of the induced map of $A^{\prime}=A / z$ into $B$, denoted again by the same letter $\pi^{\prime}$, contains neither a non-zero right-ideal nor a non-zero left-ideal in $A^{\prime}$. The map $\pi^{\prime}$, of $A^{\prime}$ into $B$, satisfies Prop. 16, $\alpha$ ) (i.e. Prop. $4, \mathrm{i}_{r}$ ) and $\mathrm{i}_{l}$ )) for $A^{\prime} . \quad A^{\prime}=A / z$ is again (finitely generated) $B$ left (or right) projective, since $z$ is a left (or right, resp.) $d$-ideal. Hence $A^{\prime}$ is $\beta$-Frobenius over $B$ by Prop. 16. (We wish to correct an error in Prop. 16: " $B$-left" in the second line in Prop. 16 should read " $B$-right" (in order to be in accord with " $\left.1_{r}\right)$ " in the third line. However, if we replace this " $1_{r}$ )" by " $1_{l}$ )", then " $B$-left" goes well (without injuring the validity of the proposition)).

Remark. The application of $L_{\pi}$ shows that the condition a') for a left $d$ ideal $z$ is weaker than the condition a).

Lemma 32. Let be 2.Frobenius over $B$. Assume that the B-right-module $A$ has a direct sumnand, say $\Re$, which can be (B-right) homomorphically mapped upon $B$; this is automatically the case with $\mathfrak{\imath}=B$ if $B$ is an S-ring. For $\pi=\emptyset 1$ as in Prop. 4 we have then $\pi A \Re=B$ and so $\pi A=B$.

Proof. There exists, by assumption, a direct $B$-right decomposition $A=\Re \oplus \Re_{1}$ of $A$ and $\Re$ has a $B$-right epimorphism $\psi$ onto $B$. Mapping $x+x_{1}$ ( $x \in \Re, x_{1} \in \Re_{1}$ ) to $\beta \psi x \in B$, where $\beta$ is an automorphism of $B$ belonging to the 2.Frobenius extension $A$ over $B$, we obtain an element $\varphi$ of $\operatorname{Hom}_{B, \beta}\left(A_{B}, B_{B}\right)$. Since $\psi$ is epimorphic, $\varphi$ is epimorphic and indeed $\varphi \Re=B$. By Prop. 4 $\dot{\phi} \in \Phi A$. So $B=(\Phi A) \Re=(\emptyset 1) A \Re=\pi A \Re$, proving the lemma.

Theorem 33. Let $A$ be a $\beta$-Frobenius extension of an S-ring $B$ and $z$ be $a$ (two-sided) ideal of $A$ which is a left d-ideal of $A$ and satisfies $z \cap B=0$. The residue-ring $A / z$ is $\beta$-Frobenius over $B$ if and only if there is an element $a$ in $A$ such that $\left.\mathrm{a}^{\prime}\right)$ of Prop. 31 and $\mathrm{b}$ ) of Prop. 30 hold, i.e. $z=L_{\pi} A a=L_{\pi} a A$ and $\pi(x y a-x a y)=0$ for all $x \in A, y \in B$, where $\pi$ is as in Prop. 4.

Proof. The "if" was proved in Proposition 31. To prove "only if", assume that the residue-ring $A^{\prime}=A / z$ is $\beta$-Frobenius over $B$. There exists then a $(B$, 1)-( $B, \beta$ )-homomorphism $\pi^{\prime}$ of $A^{\prime}$ into $B$ which has the properties $\mathrm{i}_{r}$ ), $\mathrm{i}_{l}$ ), $\mathrm{ii}_{r}$ ), $\mathrm{ii}_{l}$ ) of Prop. 4 with respect to $A^{\prime}$ in place of $A$. Let $\Omega^{\prime}$ be the kernel of $\pi^{\prime}$, i.e.

$$
\Re^{\prime}=L_{\pi^{\prime}} 1^{\prime}=R_{\pi^{\prime}} 1^{\prime}
$$

(where $1^{\prime}$ is the unit element $\left(1 \bmod z\right.$ ) of $\left.A^{\prime}\right)$. As the risidue-module $A^{\prime} / \Omega^{\prime}$ 
is $(B, 1)-(B, \beta)-$, whence $B$-left-, isomorphic to $B$, by Lemma 32 , (and $B$ is $B$-left projective), there is a $B$-left submodule $\mathscr{L}^{\prime}$ of $A^{\prime}$ with $A^{\prime}=\Re^{\prime} \Phi \mathbb{Q}^{\prime} . \quad \pi^{\prime}$ induces a $B$-left isomorphism of $\mathfrak{L}^{\prime}$ and $B$.

Now, since $z$ is a left $d$-ideal, we have $A=z \oplus \mathfrak{Q}$ with a $B$-left submodule $\mathfrak{Z}$ of $A$. By the canonical map $A \rightarrow A^{\prime}=A / \delta, \mathbb{Z}$ is mapped $B$-left isomorphically onto $A^{\prime}\left(=\mathscr{R}^{\prime} \oplus \mathfrak{L}^{\prime}\right)$. Denoting by $\mathscr{I}^{\prime \prime}$, $\mathbb{L}^{\prime \prime}$ the counter-images in $\mathfrak{L}$ of $\mathfrak{K}^{\prime}$, $\mathfrak{L}^{\prime}$, we obtain the direct $B$-left decomposition $A=\delta \oplus \dot{\mathfrak{R}}^{\prime \prime} \oplus \mathfrak{Q}^{\prime \prime} ; z \oplus \mathfrak{\Omega}^{\prime \prime}$ is the counterimage, in $A$, of $\mathfrak{I}^{\prime}$ by the map $A \rightarrow A^{\prime}=A /$. . By Prop. 6 we have the direct $B$-right decomposition

$$
A=R_{\pi}\left(\mathscr{\Omega}^{\prime \prime} \oplus \mathbb{L}^{\prime \prime}\right) \oplus R_{\pi}\left(z \oplus \mathbb{Q}^{\prime \prime}\right) \oplus R_{\pi}\left(z \oplus \mathscr{\Re}^{\prime \prime}\right) .
$$

As $R_{\pi}\left(z \oplus \mathfrak{R}^{\prime \prime}\right) \approx \operatorname{Hom}_{B, \beta^{-1}}\left(\mathcal{L}^{\prime \prime},{ }_{B} B\right)\left(B\right.$-right) and as $\mathbb{I}^{\prime \prime} \approx \mathfrak{Q}^{\prime} \approx B$ (B-left), we see $R_{\pi}\left(z \oplus \AA^{\prime \prime}\right) \approx \operatorname{Hom}_{B, \beta^{-1}}\left({ }_{B} B,{ }_{B} B\right) \approx B$ ( $B$-right $)$. Hence there is an element $a$ in $A$ with $R_{\pi}\left(z \oplus \Omega^{\prime \prime}\right)=a B$. Hence $z \oplus \mathfrak{\Omega}^{\prime \prime}=L_{\pi} a B=L_{\pi} a$, again by Prop. 6 . Now, $z$ is the largest right-ideal of $A$ contained in $\delta \oplus \mathcal{R}^{\prime \prime}$, since otherwise the kernel $\bar{\Omega}^{\prime}$ of $\pi^{\prime}$ would c nntain a non-zero right-ideal of $A^{\prime}$. So, as $\gamma \oplus \bar{\Omega}^{\prime \prime}=L_{\pi} a B=L_{\pi} a$, we obtain $z=L_{\pi} A a=L_{\pi} A a B$ by Lemma 24. Similarly $z$ is the largest left-ideal of $A$ contained in $z \oplus \AA^{\prime \prime}$ and hence, by Lemma $2 \overline{5}, z=L_{\pi} a A$.

The map $x \rightarrow \pi x a$ is a $B$-left-homomorphism of $A$ into $B$. Its kernel is the module $L_{\pi} a$ which we have just seen to be $z \oplus \AA^{\prime \prime}$. It follows that the map

$$
\left(x \bmod z \oplus \Omega^{\prime \prime}\right) \in A /\left(z \oplus \Re^{\prime \prime}\right) \rightarrow \pi x a \subseteq B
$$

is a $B$-left-isomorphism of $A /\left(z \oplus \mathscr{R}^{\prime \prime}\right)$ onto $B$. On the other hand, $A /\left(z \oplus \Omega^{\prime \prime}\right)$ $=A^{\prime} / \Re^{\prime}$ and we have a $B$-left (and even $(B, 1)-(B, \beta)$-) isomorphism of $A^{\prime} / \AA^{\prime}$ onto $B$ given by

$$
\left(x^{\prime} \bmod \AA^{\prime}\right) \rightarrow \pi^{\prime} x^{\prime} \in B .
$$

(which we have made use of before). So the composite

$$
\pi x a \rightarrow\left(x \bmod z \oplus \mathscr{\Re}^{\prime \prime}\right) \rightarrow\left((x \bmod z) \bmod \mathscr{\Re}^{\prime}\right) \rightarrow \pi^{\prime}(x \bmod z)
$$

is a $B$-left isomorphism of $\pi A a(\subseteq B)$ onto $B$. Considering $B$-left lengths, we see that $\pi A a=B$ and $\pi x a \rightarrow \pi^{\prime}(x \bmod z)$ is a $B$-left automorphism of $B$. Hence there is a regular element $b$ in $B$ such that

$$
(\pi x a) b=\pi^{\prime}(x \bmod 3) \quad \text { for all } x \in A .
$$


Since $x \rightarrow \pi^{\prime}(x \bmod z)$ is $(B, 1)-(B, \beta)$-homomorphic, we have hence $(\pi x y a) b$ $=(\pi x a) b \beta y$, i.e. $\pi x y a \beta^{-1} b=\pi x a\left(\beta^{-1} b\right) y$, for all $y \in B$ (and for all $x \in A$ ). Putting $a_{0}=a \beta^{-1} b$, we have

$$
\pi x y a_{0}=\pi x a_{0} y
$$

for all $y \in B, \quad x \in A$. As $\left.L_{\pi} A a_{0}=L_{\pi} A a_{0} B=L_{\pi} A a B=L_{\pi} A a(=\}\right), L_{\pi} a_{0} A$ $=L_{\pi} a A(=8)$, we have the "only if" part of the proposition on denoting $a_{0}$ by $a$ anew.

Remark. As our proof shows, the "only if" part of Prop. 33 remains valid when we replace the $S$-ring condition on $B$ by that $B$ satisfies the minimum condition for left -ideals and $A^{\prime}=A / z$ has a $B$-right direct summand ( $B$-right) isomorphic to $B$.

\section{$\S 10$. Frobenius extension of a quasi-Frobenius ring.}

By homological means we have seen already in Corollary 11 that $a$ 2.Frobenius extension of a quasi-Frobenius ring $B$ is a quasi-Frobenius ring. Using the annihilator characterization of quasi-Frobenius rings, instead of homological characterization, we may derive this theorem also from the following generalized formulation of Hall's [15] theorem:

Proposition 34. Let $B$ be a quasi-Frobenius ring and $\beta$ be an automorphism of $B$. Let $\mathfrak{L}$ be a finitely generated projective B-left-module, il a B-right-module, and let there be given a regular 1- $\beta$-scalar product $\langle$,$\rangle of \mathfrak{L}, \mathfrak{R}$ in $B$; then $\Re$ is finitely generated B-right projective, by Prop. 15. For every B-left submodule $\mathfrak{S}_{0}$ of $\mathfrak{Q}$ (resp. B-right submodule $\mathfrak{R}_{0}$ of $\Re$ ) we have

$$
L R \mathfrak{Q}_{0}=\mathfrak{Q}_{0} \quad\left(\text { resp. } R L \mathfrak{R}_{0}=\mathfrak{X}_{0}\right),
$$

where $L, R$ are the same as in Proposition 15, 2).

Proof. For the sake of completeness and convenience we briefly reproduce Hall's proof in the form adapted to our present generalized formation. Expressing $\mathfrak{R}$ as a direct sum of submodules isomorphic to right-ideals generated by an idempotent and applying Prop. 15 repeatedly, we may express $\mathfrak{N}, \mathfrak{\&}$ as direct sums

$$
\begin{aligned}
\mathfrak{R} & =v_{1} B \oplus \cdots \oplus v_{n} B, \\
\mathfrak{L} & =B u_{1} \oplus \cdots \oplus B u_{n}
\end{aligned}
$$


with $v_{i}, u_{\text {, such }}$ that $v, B \approx e_{2} B$ ( $B$-right $), B u_{2} \approx B \beta e_{i}$ ( $B$-left $)$ by $v_{\star} \leftrightarrow e_{i}$, $u_{*} \leftrightarrow \beta e_{\imath}, e$, being idempotents in $B$, and furthermore

$$
\left\langle u_{\mu}, v_{\nu}\right\rangle=\delta_{\mu \nu} \beta e_{\nu} .
$$

Denote by $\Re^{*}$, $\mathfrak{\Omega}^{*}$ the submodules $v_{2} B \oplus \cdots \oplus v_{n} B, B u_{2} \oplus \cdots \oplus B u_{n}$ of $\Re, \mathbb{Q}$. The scalar product $\langle$,$\rangle on \mathfrak{R}, \mathfrak{L}$ induces an (also $(1, \beta)$ ) scalar product on $\mathfrak{R}^{*}, \mathfrak{I}^{*}$, which is also regular and which we denote also by $\langle$,$\rangle . For a sub-$ module $\Re_{0}$ of $\Re$ we denote by $\Re_{0}^{*}$ the intersection $\Re_{0} \cap \Re^{*}$. Similarly we set $\mathfrak{Q}_{0}^{*}=\mathfrak{Q}_{0} \cap \mathfrak{Q}^{*}$ for a submodule $\mathfrak{Q}_{0}$ of $\mathfrak{Q}$. Now, in order to prove our proposition by induction, with respect to $n$, we assume that the assertion for $\Re^{*}, \Omega^{*}$ (and their scalar product $\langle$,$\rangle ), in place of \mathfrak{R}, \mathbb{Q},($ and $\langle$,$\rangle ), is valid. Then our$ induction argument is divided into several steps.

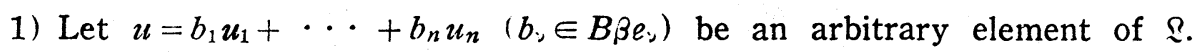
Then (23) holds for $\mathfrak{I}_{0}=B u$, i.e.

$$
L R B u=B u .
$$

To prove this, let $s_{1} u_{1}+\cdots+s_{n} u_{n} \in L R B u \quad\left(s, \in B \beta e\right.$.). Then $0=\left\langle s_{1} u_{1}+\right.$ $\left.\cdots+s_{n} u_{n}, \quad v_{1} x_{1}\right\rangle=s_{1} \beta x_{1}$ for every $x_{1} \in B$ with $b_{1} \beta x_{1}=0$. Hence $s_{1} \in \operatorname{lr} b_{1}$ $=B b_{1}$, where $l, r$ are left and right annihilator operations in the quasi-Frobenius ring $B$. So $s_{1}=t b_{1}$ with $t \in B$. We may write

$$
s_{1} u_{1}+\cdots+s_{n} u_{n}-t\left(b_{1} u_{1}+\cdots+b_{n} u_{n}\right)=s_{2}^{\prime} u_{1}+\cdots+s_{n}^{\prime} u_{n}
$$

$\left(s^{\prime}, \in B\right)$. Since this element belongs to $(L R B u)^{*}$, it belongs to $L^{*} R^{*} B\left(b_{2} u_{2}+\right.$ $\left.\cdots+b_{n} u_{n}\right)$, as we readily see, and we can write

$$
s_{2}^{\prime} u_{2}+\cdots+s_{n}^{\prime} u_{n}=t^{\prime}\left(b_{2} u_{2}+\cdots+b_{n} u_{n}\right) \quad\left(t^{\prime} \in B\right)
$$

in virtue of our induction assumption.

Now, for any element $w=b_{1} z_{1}=-\left(b_{2} z_{2}+\cdots+b_{n} z_{n}\right)\left(z_{2} \in B\right)$ common to $b_{1} B$ and the (not necessarily direct) sum $b_{2} B+\cdots+b_{n} B$, we have evidently $b_{1} z_{1}+\cdots+b_{n} z_{n}=0$ and thus $v_{1} \beta^{-1} z_{1}+\cdots+v_{n} \beta^{-1} z_{n} \in R B u$. Since $t^{\prime}\left(b_{2} u_{2}\right.$ $\left.+\cdots+b_{n} u_{n}\right)=s_{2}^{\prime} u_{2}+\cdots+s_{n}^{\prime} u_{n} \in L R B u$, this entails $t^{\prime} w=-t^{\prime}\left(b_{2} z_{2}+\cdots\right.$ $\left.+b_{n} z_{n}\right)=\left\langle s_{2}^{\prime} u_{2}+\cdots+s_{n}^{\prime} u_{n}, v_{1} \beta^{-1} z_{1}+\cdots+v_{n} \beta^{-1} z_{n}\right\rangle=0$. This shows

$$
t^{\prime} \in l\left(b_{1} B \cap\left(b_{2} B+\cdots+b_{n} B\right)\right) .
$$

The right-hand side is equal to $l b_{1} B+l\left(b_{2} B+\cdots+b_{n} B\right)$ and hence $t^{\prime}=p+q$ with $p \in l b_{1} B, g \in l\left(b_{2} B+\cdots+b_{n} B\right)$. Therefore 


$$
\begin{aligned}
& t^{\prime}\left(b_{2} u_{2}+\cdots+b_{n} u_{n}\right)=p\left(b_{1} u_{1}+b_{2} u_{2}+\cdots+b_{n} u_{n}\right)=p u \\
& s_{1} u_{1}+\cdots+s_{n} u_{n}=t u+\left(s_{2}^{\prime} u_{2}+\cdots+s_{n}^{\prime} u_{n}\right) \\
& \quad=t u+t^{\prime}\left(b_{2} u_{2}+\cdots+b_{n} u_{n}\right)=t u+p u \\
& \quad=(t+p) u \in B u .
\end{aligned}
$$

This proves $L R B u(\subseteq$ whence $)=B u$.

2) Next we show that if $u$ is an element of $\mathfrak{Q}$ then $R^{*}(B u)^{*}=\left(R B u+v_{1} B\right)^{*}$. For, $L^{*}\left(R B u+v_{1} B\right)^{*} \subseteq L R B u$ as we readily see. The right-hand side is $B u$ by the step 1). Thus $(B u)^{*} \supseteq L^{*}\left(R B u+v_{1} B\right)^{*}$, and therefore $R^{*}(B u)^{*}$ is contained in $R^{*} L^{*}\left(R B u+v_{1} B\right)^{*}$ which is in turn $\left(R B u+v_{1} B\right)^{*}$ by our induction assumption. This proves a half of our contension, and the other half, i.e. the inclusion of the other direction, is evident.

3 ) Let $\Omega_{0}$ be a $B$-left submodule of $\&$. Then we wish to prove $R^{*} \Omega_{0}^{*}$ $=\left(R \Omega_{0}+v_{1} B\right)^{*}$.

Again the inclusion $\subseteq$ is evident, and so we have to prove $\supseteq$, i.e. that for any element $v^{*}$ of $R^{*} \Omega_{0}^{*}$ there exists an element $x_{1}$ of $e_{1} B$ such that

$$
v_{1} x_{1}+v^{*} \in R \Omega_{0}
$$

For this purpose, let

$$
u^{(\rho)}=b_{1}^{(\rho)} u_{1}+\cdots+b_{n}^{(\rho)} u_{n} \quad\left(b_{\nu}^{(\rho)} \in B \beta e_{\nu}\right)(\rho=1, \ldots, r)
$$

be a (finite) system of $B$-left generators of $\mathfrak{L}_{0}$. Let $s \leqq r$ and assume that there is an $x_{1}^{\prime} \in e_{1} B$ satisfying

$$
\left\langle u^{(\rho)}, v_{1} x_{1}^{\prime}+v^{*}\right\rangle=0 \quad \text { for } \rho=1, \ldots, s-1 .
$$

By the above step 1) there is $x_{1}^{\prime \prime} \in e_{1} B$ satisfying

$$
\left\langle u^{(s)}, v_{1} x_{1}^{\prime \prime}+v^{*}\right\rangle=0 .
$$

Now, let $w=y^{(1)} b_{1}^{(1)}+\cdots+y^{(s-1)} b_{1}^{(s-1)}=y^{(s)} b^{(s)}\left(y^{(\sigma)} \in B\right)$ be an element of $\left(B b_{1}^{(1)}+\cdots+B b_{1}^{(s-1)}\right) \cap B b_{1}^{(s)}$. Then

$$
y^{(1)} u^{(1)}+\cdots+y^{(s-1)} u^{(s-1)}-y^{(s)} u^{(s)} \in \mathbb{R}_{0}^{*} .
$$

Hence

$$
\left\langle y^{(1)} u^{(1)}+\cdots+y^{(s-1)} u^{(s-1)}-y^{(s)} u^{(s)}, \quad v^{*}\right\rangle=0 .
$$

In combination with (25), (26) this entails 


$$
\begin{gathered}
\left\langle y^{(1)} u^{(1)}+\cdots+y^{(s-1)} u^{(s-1)}, v_{1} x^{\prime}\right\rangle-\left\langle y^{(s)} u^{(s)}, v_{1} x_{1}^{\prime \prime}\right\rangle=0, \text { i.e. } \\
w \beta x_{1}^{\prime}-w \beta x_{1}^{\prime \prime}=w \beta\left(x_{1}^{\prime}-x_{1}^{\prime \prime}\right)=0 .
\end{gathered}
$$

We have thus seen

$$
\begin{aligned}
\beta\left(x_{1}^{\prime}-x_{1}^{\prime \prime}\right) \in r\left(\left(B b_{1}^{(1)}+\cdots+B b_{1}^{(s-1)}\right) \cap B b_{1}^{(1)}\right)= \\
r\left(B b_{1}^{(1)}+\cdots+B b_{1}^{(s-1)}\right)+r B b_{1}^{(1)} .
\end{aligned}
$$

Hence $\beta\left(x_{1}^{\prime}-x_{1}^{\prime \prime}\right)=p+q$ with

$$
p \in r\left(B b_{1}^{(1)}+\cdots+B b_{1}^{(s-1)}\right), \quad q \in r B b_{1}^{(1)} .
$$

Put $x_{1}^{\prime \prime \prime}=x_{1}^{\prime}-\beta^{-1} p\left(=x_{1}^{\prime \prime}+\beta^{-1} q\right)$. Then by (25), (26) and (27)

$$
\left\langle u^{(\rho)}, v_{1} x_{1}^{\prime \prime \prime}+v^{*}\right\rangle=0 \quad \text { for } \rho=1, \ldots, s .
$$

$(25) \rightarrow(28)$ makes $s-1 \rightarrow s$ and we reach to our contension by recursion of this argument.

4) Let (24) be as in the preceding step, and $u=y_{1} u_{1}+\cdots+y_{n} u_{n}$ ( $y_{\nu}$ $\left.\in B \beta e_{\nu}\right)$ be any element of $L R \Omega_{0}$. If $x_{1}$ is an element of $B$ such that $b_{1}^{(\rho)} \beta x_{1}$ $=0$ for $\rho=1, \cdots, r$, then $v_{1} x_{1} \in R \mathfrak{Q}_{0}$ and hence $y_{1} \beta x_{1}=0$. Thus $y_{1} \in l\left(r b_{1}^{(1)} \cap\right.$ $\left.\cdots \cap r b_{1}^{(r)}\right)=\operatorname{lr} b_{1}^{(1)}+\cdots+\operatorname{lr} b_{1}^{(r)}=B b_{1}^{(1)}+\cdots+B b_{1}^{(r)}$, whence

$$
y_{1}=p^{(1)} b_{1}^{(1)}+\cdots+p^{(r)} b_{1}^{(r)} \quad\left(p^{(p)} \in B\right)
$$

The element $u^{\prime}=p^{(1)} u^{(1)}+\cdots+p^{(r)} u^{(r)}$ of $\mathfrak{L}_{0}$ has a form

$$
y_{1} u_{\mathrm{i}}+y_{2}^{\prime} u_{2}+\cdots+y_{n}^{\prime} u_{n} \quad\left(y_{\nu}^{\prime} \in B \beta e_{\nu} ;, \nu=2, \ldots, n\right) .
$$

We have $u-u^{\prime} \in\left(L R \Omega_{0}\right)^{*}$ and hence

$$
\left\langle u-u^{\prime}, v^{*}\right\rangle=0
$$

for every $v^{*} \in\left(R \Omega_{0}+v_{1} B\right)^{*}$, whence, by the preceding step 3$)$, for every $v^{*} \in R^{*} \mathfrak{L}^{*}$. So $u-u^{\prime} \in L^{*} R^{*} \mathfrak{Q}_{0}^{*}$ and $\in \mathfrak{Q}_{0}^{*}$ by our induction assumption. Hence

$$
u=u^{\prime}+\left(u-u^{\prime}\right) \in \mathfrak{Q}_{0}+\mathfrak{Q}_{0}^{*}=\mathfrak{\Omega}_{0} .
$$

This completes our final step, proving thus Proposition 34. Now, as a second application of Prop 34, Hall's theorem (in generalized formulation), we combine it with Prop. 30 and Theorem 33 to obtain

Theorem 35. Let $A$ be a $\beta$-Frohenius extension of a quasi-Froebenius ring $B$ and 3 be a (two-sided) ideal of $A$ which is a left d-ideal of $A$ and satisfies 
$\hat{\jmath} \cap B=0$. The residue-ring $A / z$ is $\beta$-Frobenius over $B$ if and only if there is an element $a$ in $A$ such that $r=A a B=a A$ and $\pi(x y a-x a y)=0$ for all $x \in A$, $y \in B$, where $\pi$ is as in Prop. 4.

Proof. If $r \hat{\jmath}=A a B=a A$, then, since $\left.r \hat{\jmath}=R_{\pi}\right\}, \jmath\left(=L_{\pi} R_{\pi} \hat{\jmath}\right)=L_{\pi} A a B\left(=L_{\pi} A a\right)$ $=L_{\pi} a A$. Hence the "if" part of our theorem follows from Prop. 30. To prove the "only if", we have merely to note that the condition $z=L_{\pi} A a=L_{\pi} a A$ of Theorem 33 entails $R_{\pi} z(=r z)=R_{\pi} L_{\pi} a B=R_{\pi} L_{\pi} a A$ and to observe that here $R_{\pi} L_{\pi} A a B=A a B, R_{\pi} L_{\pi} a A=a A$ in virtue of Prop. 34 .

Remark. Our theorem fails to cover Satz 9 of Kasch [10]; particulary, our by-condition $\pi(x y a-x a y)=0$ is very strong. However, the writers fail to convince themselves of the validity of the proof of [10], Satz 9 .

\section{References (some repeating ones in Part I)}

[4] S. Eilenberg-T. Nakayama, On the dimension of modules and algebras II, Nagoya Math. J. 9 (1955), 1-16.

[6] K. Hirata, On relative homological algebra of Frobenius extensions, Nagoya Math. J. 15 (1959), 17-28.

[10] F. Kasch, Grundlagen einer Theorie der Frobeniuserweiterungen, Math. Ann. 127 (1954), 453-474.

[13] T. Nakayama- T. Tsuzuku, A remark on Frobenius extensions and endomorphism rings, Nagoya Math. J. 15 (1959), 9-16.

[14] C. Curtis, Quasi-Frobenius rings and Galois theory, Ill. J. Math. 3 (1959), 134-144.

[15] M. Hall, A type of algebraic closure, Ann. Math. 40 (1939), 360-369.

[16] M. Ikeda, On a theorem of Gaschütz, Osaka Math. J. 5 (1953), 53-58.

[17] T. Nakayama, On Frobeniusean algebras I, Ann. Math. 40 (1939), 611-633.

[18] T. Nakayama, Galois theory of simple rings, Trans. Amer. Math. Soc. 73 (1952), 276-292.

\section{Mathematical Institute}

Nagoya University 